\title{
Evaluación del Impacto del Modelo Acción Docente como Alternativa Pedagógica para el Manejo del Trastorno Disocial Escolar desde la Perspectiva Humanística
}

\author{
Jesús A. Gómez \\ Universidad de Otavalo, Carrera de Derecho. Av, Sarances y los Pendoneros, Otavalo, Ecuador. \\ (e-mail: jgomez@uotavalo.edu.ec; jesgomez35@gmail.com)
}

Recibido Feb. 28, 2018; Aceptado May. 9, 2018; Versión final Jun. 21, 2018, Publicado Dic. 2018

\begin{abstract}
Resumen
Se presentan los resultados de un estudio diseñado para evaluar el impacto del modelo acción docente como alternativa pedagógica para el manejo del trastorno disocial escolar, fundamentado en la perspectiva humanístic. El estudio está dirigido a los docentes de Educación Secundaria de la Unidad Educativa Alto Barinas Norte, en Venezuela. Este tipo de trastorno se manifiesta en adolescentes en la etapa de educación secundaria cuyos comportamientos son de tipo violento y agresivo, y que infringen las normas de convivencia escolar. La metodología empleada fue de tipo proyectivo que desarrolló la planificación, ejecución y evaluación del modelo. Se utilizó como instrumento la escala tipo Likert aplicada por los 3 directivos del plantel con el propósito de establecer los resultados y el impacto del modelo tras su ejecución. Como resultado, se confirmó la participación y aceptación los docentes para orientar la conducta estudiantil. En conclusión, la ejecución del modelo fortaleció la acción docente y las competencias para manejar este tipo de trastorno.
\end{abstract}

\section{Evaluation of the Impact of the Teaching Action Model as a Pedagogical Alternative for the Management of School Dissocial Disorder from a Humanistic Perspective}

\begin{abstract}
The results of a study designed to evaluate the impact of the teaching action model as a pedagogical alternative for the management of school dissocial disorder, based on the humanistic perspective is presented. The study is aimed at Secondary Education teachers of the Alto Barinas Norte Educational Unit in Venezuela. School dissocial disorder is manifested in adolescents in the stage of secondary education having violent and aggressive behavior, violating the rules of school coexistence. The methodology used was of projective type that developed the planning, execution and evaluation of the model. A Likert-type scale applied by the 3 managers of the school was used for establishing the results and the impact of the model after its execution. As a result, the participation and acceptance of teachers to guide student behavior was confirmed. In conclusion, the execution of the model strengthened the teaching action and the competences to handle this type of disorder.
\end{abstract}

Keywords: educational model; teaching action; pedagogical alternative; dissocial school disorder. 


\section{INTRODUCCIÓN}

El modelo de acción docente es una alternativa para el manejo del trastorno disocial escolar Gómez, (2018) y Palella y Martins, (2012), es un esquema epistemológico que representa la realidad de una institución educativa relacionada con el desempeño docente y el comportamiento del estudiante. Además, aborda temáticas vinculadas con la acción docente, competencias, ejercicio de roles y estrategias aplicadas al proceso educativo para prevenir comportamientos disociales. Con base a este idea Wang y Fredricks, (2014) señalan desde décadas atrás se ha venido estudiando la conducta de los jóvenes entre 12 y 16 años, donde explican hechos relacionados con el comportamiento disocial concernientes con la agresividad, la violencia, el robo, incumplimiento a la norma que afecta el bienestar y la formación integral del joven estudiante. Al respecto Urhahne, (2015) demuestra en la literatura el docente puede realizar acciones bajo este contexto que motiven al estudiante a prevenir estos comportamientos y mejorar así el rendimiento académico.

En el caso de Venezuela, Abbott y Vom Hau (2017) y Vera, et al., (2017) manifiestan que las instituciones educativas enfrentan hoy en día cambios económicos, políticos, culturales que afectan la vida del estudiante y su desenvolvimiento personal. Esta situación según Acevedo et al. (2015) y Urazán-Torres et al., (2013) conlleva a crear en el joven un déficit de valores surgimiento de nuevos comportamientos agresivos que violan la norma y transgresiones a los derechos que afectan la conducta del adolescente. Sin embargo, Vera et al., (2013) expresa a través de un estudio de tesis doctoral "Conducta Antisocial: Asociación con Psicopatología en Niños y Adolescentes" tuvo como propósito estudiar la prevalencia y la asociación diferencial de las conductas antisociales con una mayor gravedad de los problemas de salud mental. Concluyó que las conductas antisociales en adolescentes, son altamente prevalentes, constituyen una de las principales causas de consultas recurrentes a centros de salud mental, generan diversas dificultades sobre el funcionamiento cotidiano de los individuos y su entorno social. El estudio se fundamentó bajo el paradigma cualitativo y los métodos usados fueron el modelo de regresión logística, binomial-negativa, múltiple con ecuaciones estructurales. El resultado de este estudio coincide con el de Piotrowska, et al. (2015) quienes afirman que la presencia de conductas antisociales en los jóvenes en edades comprendidas entre los 13 y 17 años de edad, está asociada mayormente a problemas relacionados con el sexo y la sobreprotección por parte de los padres, quienes aceptaban en ellos este tipo de comportamiento. La presencia de conductas antisociales no difirió significativamente según el sexo o la edad de los individuos, no obstante, se observó una prevalencia mayor de estas conductas entre los 13 y los 17 años, y en el sexo masculino, estableció como fundamental la valoración de las conductas antisociales en escolares cuyas edades oscilen entre dichos límites.

En referencia a estos hechos, la Unidad Educativa "Alto Barinas Norte" ubicada en la ciudad de Barinas, parroquia Alto Barinas, municipio Barinas del estado Barinas, Venezuela, se observó una situación similar vinculada con este tipo de trastorno a nivel individual y grupal en los jóvenes estudiante de educación secundaria. Tal es el caso, Ornelas et al., (2012); Cheon, et al., (2018) manifiestan la presencia de agresividad entre los mismos estudiantes involucran a miembros de otros niveles de la institución, poniéndose de manifiesto amenazas, agresiones físicas y el incumplimiento a las normas de convivencia escolar, considerando las definiciones dadas de trastorno disocial, tales expresiones conductuales fueron asumidas como síntomas o probables manifestaciones de este tipo. En tal sentido, la acción docente Bradfield et al., (2015), se refiere a la enseñanza apoyada en el aprendizaje acción. De ahí, el modelo tiene como eje fundamental el desarrollo de talleres de capacitación docente que buscan promover experiencias y procedimientos contribuyen con el desarrollo de competencias para el manejo de conductas relacionadas con el trastorno disocial escolar en los estudiantes de educación secundaria de la Unidad Educativa "Alto Barinas Norte". Además, como herramienta beneficia directamente a los docentes de la institución mediante la preparación para desempeñar con eficacia la práctica pedagógica humanista, basada en competencias que permitan el manejo de este tipo de trastorno en el aula de clase. Sin embargo, Rosa et al., (2015) afirma que la acción pedagógica en el docente busca promover en el estudiante situaciones significativas que contribuyan con su formación integral. Así que, a través de este modelo se orienta el comportamiento hacia el logro de los objetivos de aprendizaje, el cumplimiento de las normas de convivencia escolar y el reconocimiento de la importancia de los valores humanistas para la vida. Así como también Flores et al., (2012) coinciden que el profesor desarrolla las habilidades cognitivas, competencias personales, valores, así como estrategias de gestión de las emociones para actuar conforme a la realidad educativa, ofrecer las asistencia personal que proporcione al educando la seguridad y la motivación para mejorar el comportamiento dentro y fuera de la institución educativa.

Por su parte, el modelo se fundamenta en la teoría de la acción docente de Talcott Parsons, Almeida, (2001); Contreras, (2009), su enfoque se orienta hacia la acción pedagógica como un hecho humano visible, tangible, sistemático, ejercido por el docente con el objetivo primordial de llevar a cabo un proceso de interacción de enseñanza aprendizaje y lograr la formación integral del educando. Bontempi y Pietri, (2017) demuestran la acción docente tiene como responsabilidad promover experiencias de aprendizaje, desarrollar tareas, tomar decisiones que contribuyan en el logro de las metas del estudiante, la construcción del conocimiento, 
habilidades que faciliten el manejo de la conducta, especialmente de aquellas relacionadas o proclives a comportamientos disóciales, a partir de la disminución de la agresividad y la mejora de la armonía en el plantel educativo, mediante el cumplimiento de las normas de convivencia escolar. Aunado a este aspecto, Vieira y Pinheiro, (2013), expresan que la teoría de Carl Rogers, se basa en el enfoque centrado en la persona, las relaciones humanas, basado en lo que el determinó como la persona plenamente funcional, la conducta propia, valora la armonía entre el hombre y el yo. Al igual, Dutra et al., (2016), declaran esta teoría se fundamenta en la psicología humanista donde asume como preferencia la experiencia, el comportamiento, la subjetividad de la persona según su mundo interior, los valores y las relaciones interpersonales.

Siguiendo este planteamiento, Bryan et al., (2015), aseveran que el enfoque centrado en la persona de Carl Rogers, el profesional es un educador que puede influenciar en el comportamiento del estudiante dentro de la institución mediante la formación de las relaciones humanas. De ahí, Zalyaeva y Solodkova, (2014), ratifican el docente utiliza distintos roles que se adecúan a las necesidades del joven estudiante, así como en situaciones específicas que desempeña una función clave el procesos de enseñanza aprendizaje. Asimismo, Todorescu et al., (2015); Blašková et al., (2014); Smolkowski, et al., (2017), aseguran, el docente puede ejercer el rol de orientador en el ambiente escolar y la familia, cuya autoridad intelectual permite aconsejar, asesorar el comportamiento estudiantil, como agente de cambio, líder y facilitador que brinda apoyo en la institución educativa. De lo antes descrito, el docente en su rol como orientador, se apoya en la capacidad propia de cada individuo para fortalecer las relaciones interpersonales, fomentar el cumplimiento de las normas de convivencia y despierta el interés en el estudiante por los valores humanos como lo es el respeto a los demás como personas. Razón por la cual, Vieira y Pinheiro, (2013), concibe la perspectiva humanista como el estudio centrado en la persona de la teoría de Carl Rogers, así como también el comportamiento, las relaciones interpersonales que garantizan la armonía entre el individuo y su ser interior, Pérez, (2011); Contreras, (2009) expresan el modelo de acción docente propuesto, se enfoca en el humanismo el cual facilita la interacción entre el orientador y el orientado, el docente como orientador promueve un proceso de ayuda, asesoría, consejería y un ambiente de interacción positiva para generar un buen comportamiento en el estudiante. En efecto, este modelo surge como una alternativa del modelo pedagógico psicométrico existente en la institución educativa, considerado rígido, tradicionalista y limitado para formular datos.

En efecto, Carvalho y Novo, (2014), confirman la necesidad de implementar en la institución un programa o modelo que ayude al docente establecer estrategias que faciliten al estudiante una adecuada orientación individual y una buena conducta en el ambiente escolar. De allí, Vieira y Pinheiro, (2013) señalan la relación que existe entre la teoría de Carl Rogers y el rol del docente como orientador desempeña un papel clave en la vida del adolescente, ayuda al mismo a ser electivo, capaz de adoptar su propio comportamiento para lograr metas de vida, a ser responsable en las decisiones, actos volitivos que reflejan en su correcta acción. En consecuencia, un modelo de acción docente fundamentado en la perspectiva humanista, centrado en el estudiante, representa según Aarón, et al., (2016), un esquema epistémico referido a conceptos teóricos, estrategias, relacionados con hechos reales que proporciona respuesta a la situación problemática abordada. Cid, et al., (2017), el modelo se asienta en la teoría centrada en la persona de Carl Roger y la acción docente desde la teoría de Talcott Parsons, que proporciona elementos clave para fortalecer la gestión docente desde el aspecto humanista. Por tanto, el presente estudio aborda las siguientes preguntas de investigación: ¿Qué tipo de comportamientos disóciales existen en los estudiantes de educación secundaria en la institución objeto de estudio? ¿Qué características y elementos componen el modelo de acción docente como alternativa para el manejo del trastorno disocial escolar en los estudiantes de secundaria del plantel en cuestión?. Por consiguiente, ¿Cuáles son las fases de planificación para el modelo de acción que facilite el manejo del trastorno disocial escolar dirigido a los docentes de Educación Secundaria? ¿Cómo lograr la ejecución del modelo de acción docente para el manejo del trastorno disocial escolar desde la perspectiva humanista dirigido a los docentes de la institución objeto de estudio?. Por último, ¿Cómo evaluar el impacto del modelo de acción luego de su aplicación en los docentes de secundaria de la Unidad Educativa "Alto Barinas Norte"?

En referencia a las preguntas antes planteadas, se establece como objetivo general: Evaluar el impacto sobre el modelo de acción como alternativa pedagógica para el manejo del trastorno disocial escolar desde la perspectiva humanística, dirigido a los docentes de secundaria de la Unidad Educativa "Alto Barinas Norte", ubicada en la parroquia Alto Barinas, municipio Barinas estado Barinas, Venezuela. Como objetivos específicos: Analizar el comportamientos disóciales existente en el estudiante de educación secundaria en la Unidad Educativa "Alto Barinas Norte", ubicada en la parroquia Alto Barinas municipio Barinas estado Barinas, Venezuela. Describir el modelo de acción docente como alternativa para el manejo del trastorno disocial escolar desde la perspectiva humanista dirigido a los docentes de educación secundaria de la Unidad Educativa "Alto Barinas Norte". Así mismo, planificar las fases para desarrollar el modelo de acción para el manejo del trastorno disocial escolar dirigido a los docentes de educación secundaria. Ejecutar el modelo de acción docente para el manejo del trastorno disocial escolar desde la perspectiva humanista dirigido a los docentes de la institución objeto de estudio y por ultimo evaluar el impacto del modelo de acción luego de su aplicación. 


\section{MATERIALES Y MÉTODOS}

La metodología del estudio se fundamentó en la investigación proyectiva, Palella y Martins, (2012); Gómez, (2018) consistió en la planificación, ejecución y evaluación de una propuesta que respondió a la necesidad de un modelo epistémico sustentado en la teoría de acción docente facilitando el manejo del trastorno disocial escolar. Para medir el impacto del modelo, Hernández et al., (2014); Bastos et al., (2014) se aplicó un instrumento observación de tipo Escala Likert dirigidos a los diecinueve docentes (19) muestra del estudio con el propósito principal de registrar las experiencias, satisfacciones y aceptación de las temáticas ejecutadas en cada fase. Los resultados fueron validados mediante el coeficiente de confiabilidad Alfa de Cronbach, Méndez y Monescillo, (2009); García y Suárez (2013); Jorm, (2015), la validez es el grado en que los resultados del estudio reflejaron una imagen clara, representativa de la realidad observada, contrastada en un $0,87 \%$ de pertenencia y consistencia de los datos logrados.

\section{PLANIFICACIÓN DE LAS FASES DEL MODELO DE ACCIÓN DOCENTE}

El modelo se estructuró en cinco fases basadas en el desarrollo de talleres dirigidos a los docentes de secundaria para la formación de competencias sustentada en el enfoque humanista. Marinho-Araujo y Almeida, (2016), señalan que este tipo de competencias, son las habilidades, destrezas que posee el docente para colocar en práctica conocimientos a fin de reconocer el comportamiento disocial, solucionar conflictos y prevenir disrupciones que se puedan presentar en el aula de clase. A continuación se presenta la tabla referida a la planificación de las fases del modelo de acción docente:

Tabla 1: Fase de la Planificación del Modelo de Acción docente

Propósito Principal: Fortalecer la acción docente desde la perspectiva humanista mediante talleres para la formación en competencias que faciliten el manejo del trastorno disocial escolar en los estudiantes de secundaria, de la Unidad Educativa Alto Barinas Norte, ubicada en la parroquia Barinas, del municipio Barinas del estado Barinas.

$\begin{array}{cl}\text { Fase } & \text { Propósito de la fase } \\ \text { I } \begin{array}{l}\text { Proporcionar a los docentes de educación secundaria conocimientos teóricos sobre el comportamiento } \\ \text { disocial escolar, mediante talleres de capacitación, que favorezcan el análisis y comprensión de este tipo de } \\ \text { conducta y sus efectos en el desempeño académico estudiantil. }\end{array}\end{array}$

Capacitar a los docentes de educación secundaria mediante talleres, sobre estrategias para el desempeño de

II los roles de docente y orientador, en relación con el manejo del comportamiento disocial escolar, a fin de configurar una acción docente integradora desde la perspectiva humanista.

Caracterizar las competencias del docente para el manejo del comportamiento agresivo y delictivo en el estudiante de educación secundaria, a partir del estudio de casos y la simulación en talleres dirigidos hacia la identificación de fortalezas individuales y colectivas a desarrollar para la actuación asertiva como docente y orientador frente a situaciones de trastorno disocial.

IV Evaluación y seguimiento del desempeño docente en situaciones identificadas con el trastorno disocial desde la perspectiva humanista.

Generar procesos de autoevaluación, coevaluación y evaluación, como estrategia para la consolidación de

$\mathrm{V}$ aprendizajes mediante experiencias significativas en el ejercicio del rol de docente y rol orientador en el manejo de conductas vinculadas con el trastorno disocial. A continuación, la descripción de cada fase

Cada fase tiene un objetivo que se articula con los objetivos del modelo, a fin de fortalecer la acción docente desde la perspectiva humanista mediante talleres para la formación en competencias que faciliten el manejo del trastorno disocial escolar en los estudiantes de secundaria de la Unidad Educativa Alto Barinas Norte. Además cada fase del modelo de acción docente, se fundamenta desde el punto de vista filosófico en la Ley Orgánica de Educación, (2009), donde se establece la formación del ciudadano o ciudadana que se desea con base a las aspiraciones y expectativas de la sociedad venezolana. De esta manera, se infiere que el Subsistema Educativo de Educación Secundaria necesita profesionales de la docencia competentes para asumir las distintas situaciones de aprendizaje y conducta presentes en el ambiente áulico de forma tal que las instituciones puedan contribuir a la adquisición de destrezas y habilidades por parte del estudiante, con la finalidad de promover su desarrollo integral. En este sentido Lima, et al., (2017) el modelo de acción docente está dirigido a impulsar el potencial creativo humano, a partir de la consideración del docente como un ser social, único, capaz de asumir derechos y deberes en un colectivo social, a favor de una formación integral que enfatice los valores de libertad, honestidad, colaboración, responsabilidad y respeto; para lograr de esta forma propiciar la cooperación, el amor al trabajo, la convivencia, la paz y la armonía entre las personas, Por ejemplo, se presenta la planificación de la Fase I: 
Tabla 2: Fase I. Taller El comportamiento escolar y el trastorno disocial

Finalidad: Proporcionar a los docentes de educación secundaria conocimientos teóricos sobre el comportamiento disocial escolar, mediante talleres de capacitación, que favorezcan el análisis y comprensión de este tipo de conducta y sus efectos en el desempeño académico estudiantil

\begin{tabular}{|c|c|c|c|c|c|}
\hline Contenido & $\begin{array}{l}\text { Estrategias } \\
\text { metodológicas }\end{array}$ & Actividad & Recursos & Evaluación & Responsables \\
\hline $\begin{array}{l}\text { La conducta humano } \\
\text { El trastorno disocial } \\
\text { escolar } \\
\text { Clasificación del } \\
\text { Trastorno disocial } \\
\text { Escolar } \\
\text { La agresividad } \\
\text { escolar } \\
\text { Actos delictivos } \\
\text { Normas de } \\
\text { convivencia. } \\
\text { Incidencia del } \\
\text { trastorno disocial } \\
\text { escolar en el } \\
\text { aprendizaje }\end{array}$ & Exposición & $\begin{array}{l}\text { Presentación del taller, } \\
\text { objetivo y contenidos. } \\
\text { Intercambio sobre } \\
\text { expectativas del taller. } \\
\text { Relato de experiencias } \\
\text { vividas por parte del docente } \\
\text { en relación al tema. } \\
\text { Clase magistral sobre el } \\
\text { trastorno disocial escolar y su } \\
\text { incidencia en el aprendizaje } \\
\text { Lectura de material impreso } \\
\text { sobre el tema } \\
\text { Elaboración de cuadro } \\
\text { comparativo sobre los tipos } \\
\text { de trastorno en el ámbito } \\
\text { escolar a partir del material } \\
\text { impreso } \\
\text { Presentación de producto en } \\
\text { plenaria } \\
\text { Conclusiones y cierre de } \\
\text { experiencia }\end{array}$ & $\begin{array}{l}\text { Humanos: } \\
\text { Docentes } \\
\text { Directivos } \\
\text { Facilitadores } \\
\text { Materiales: } \\
\text { Guías } \\
\text { Papel bond } \\
\text { Borrador } \\
\text { Marcadores } \\
\text { Sillas } \\
\text { Mesas } \\
\text { Cinta } \\
\text { adhesiva } \\
\text { Aula } \\
\text { Laptop } \\
\text { Video beam }\end{array}$ & $\begin{array}{l}\text { Coevaluación } \\
\text { Intercambio } \\
\text { de opiniones } \\
\text { sobre } \\
\text { productos del } \\
\text { trabajo grupal } \\
\text { Evaluación } \\
\text { Valoración } \\
\text { cualitativa del } \\
\text { taller }\end{array}$ & $\begin{array}{l}\text { Unidad } \\
\text { administrativa } \\
\text { y personal } \\
\text { encargado de } \\
\text { administrar la } \\
\text { ejecución del } \\
\text { modelo de } \\
\text { acción docent }\end{array}$ \\
\hline
\end{tabular}

\section{EJECUCIÓN DE LAS FASES DEL MODELO}

El estudio desarrolló la ejecución de cada una de las fases con el objetivo principal de fortalecer la acción docente mediante talleres que permitieron la formación en competencias para el manejo del trastorno disocial escolar en los estudiantes de secundaria de la Unidad Educativa "Alto Barinas Norte". En efecto, Palella y Martins, (2012) la ejecución de las fases, se llevaron a cabo mediante actividades que hicieron énfasis en la necesidad de mejorar la acción docente a partir de sus competencias, habilidades para el manejo de comportamiento disocial escolar; estrategias, actividades, recursos, y evaluación. Cada fase, según Zalyaeva y Solodkova, (2014), tuvo como punto de partida, reconocer la necesidad existente en el docente con la finalidad de desarrollar las competencias en los educadores, como orientador de la conducta para prevenir comportamientos inadecuados en el aula de clase, como es el caso hechos relacionados con la agresividad escolar, actos delictivos y el incumplimiento de las normas de convivencia escolar. En este sentido, Ornelas et al., (2012), a lo largo de la ejecución de la propuesta se proporcionó al docente los conocimientos teóricos sobre este tipo de comportamiento, sus roles, competencias requeridas y estrategias, todo sustentado en la perspectiva humanista, para promover en el educando los valores que permitieron prevenir los comportamiento disóciales y contribuir con el cumplimiento de las normas de convivencia escolar.

En la ejecución de la fase I denominada: El comportamiento escolar y el trastorno disocial, tuvo como objetivo proporcionar a los docentes de educación secundaria conocimientos teóricos sobre el comportamiento disocial escolar, mediante talleres de capacitación que favorecieron el análisis y comprensión de este tipo de conducta y sus efectos en el desempeño académico estudiantil Marinho-Araujo y Almeida, (2016). La misma se inició a través de un compartir de experiencias, vivencias inquietudes, que diariamente el docente se encuentra en el ambiente escolar relacionadas con el comportamiento disocial. Posterior a ello, esta situación permitió a la ponente explicar mediante láminas expositivas, la temática vinculada con la conducta humana, tipos de trastornos, el trastorno disocial escolar y su incidencia en el aprendizaje escolar. La docente organizó a los docentes de secundaria en grupo de trabajo con el propósito de elaborar un cuadro comparativo sobre los tipos de comportamiento disocial, apoyado en la referencia bibliográfica sugerida y el intercambio de ideas. Este momento según Gómez, (2018), facilito al docente de secundaria conocer el trastorno disocial como un fenómeno que afecta la vida del educando mediante comportamiento inapropiados que se evidencia mediante la agresividad, la violencia, actos delictivos que conducen al incumplimiento de las normas de convivencia escolar. Finalmente, el cierre de esta fase consistió en seleccionar un relator para explicar la temática abordada, establecer contrastación con la realidad que vive el plantel y el trastorno disocial, identificar los comportamientos vinculados con este trastorno, generar las conclusiones y las orientaciones pertinentes, sustentadas en la teoría humanista que busca promover el desarrollo el valor intrínseco y los valores en el educando Almeida, (2001); Contreras, (2009). 


\section{RESULTADOS DE LA EJECUCIÓN DE LAS FASES}

El presente apartado está referido a los datos obtenidos del instrumento de observación escala tipo Likert aplicado a los diecinueves (19) docentes de educación secundaria de la Unidad Educativa "Alto Barinas Norte", con el propósito de establecer el impacto respectivo del modelo luego de su ejecución, Hernández, et al., (2014), el cual se describe a continuación:

Tabla 3: Dimensión conocimientos teóricos sobre el comportamiento disocial escolar

\begin{tabular}{|c|c|c|c|c|c|c|c|c|c|}
\hline \multicolumn{2}{|r|}{ Aspecto a Evaluar } & \multicolumn{2}{|c|}{$\begin{array}{l}\text { Muy de } \\
\text { acuerdo }\end{array}$} & \multicolumn{2}{|c|}{ De acuerdo } & \multicolumn{2}{|c|}{$\begin{array}{l}\text { Ni de acuerdo } \\
\text { ni en } \\
\text { desacuerdo }\end{array}$} & \multicolumn{2}{|c|}{$\begin{array}{c}\text { En } \\
\text { desacuerdo }\end{array}$} \\
\hline Ítems & $\begin{array}{l}\text { Dimensión; Conocimientos teóricos sobre el } \\
\text { comportamiento disocial escolar. }\end{array}$ & $F$ & $\%$ & $F$ & $\%$ & $F$ & $\%$ & $F$ & $\%$ \\
\hline 1 & $\begin{array}{l}\text { El trastorno disocial escolar es un patrón de } \\
\text { comportamientos repetitivos inadecuados } \\
\text { relacionados con el delito, el robo y la violación del } \\
\text { derecho a los demás. }\end{array}$ & 12 & 63 & 4 & 21 & 2 & 11 & 1 & 5 \\
\hline 2 & $\begin{array}{l}\text { Los conocimientos adquiridos sobre el trastorno } \\
\text { disocial permite al docente proporcionar una } \\
\text { orientación eficiente en el estudiante. } \\
\text { El manejo de las teorías sobre el trastorno disocial }\end{array}$ & 15 & 79 & 2 & 11 & 1 & 5 & 0 & 0 \\
\hline 3 & $\begin{array}{l}\text { escolar facilita la identificación comportamiento } \\
\text { inadecuados y sus efectos en el desempeño } \\
\text { académico estudiantil. }\end{array}$ & 12 & 63 & 4 & 21 & 2 & 11 & 1 & 5 \\
\hline 4 & $\begin{array}{l}\text { La formación basada en competencias } \\
\text { profesionales refuerza en el docente el desarrollo de } \\
\text { estrategias para la prevención del comportamiento } \\
\text { disocial en el ambiente escolar. }\end{array}$ & 10 & 53 & 6 & 32 & 2 & 11 & 1 & 5 \\
\hline
\end{tabular}

En la Tabla 3, dimensión conocimientos teóricos sobre el comportamiento disocial escolar, en el ítems 1, la mayoría de los docentes un sesenta y tres por ciento (63\%) manifestaron estar muy de acuerdo en que el trastorno disocial escolar representa un conjunto de patrones de conductas repetitivos, constantes, desadaptado que se encuentra vinculado con el delito y la vulneración del derecho a las persona, mientras una minoría, un cinco por ciento (5\%) señaló estar en desacuerdo en este aspecto. Seguidamente el ítems 2 , el setenta y nueve por ciento $(79 \%)$ expresó este tipo de conocimientos proporciona al docente la destreza para desarrollar con validez la orientación educativa en el estudiante, un cinco por cierto (5\%) manifestó estar ni de acuerdo ni en desacuerdo sobre esta idea. Al igual que el ítems 3, la mayor parte de los profesores un sesenta y tres por ciento (63\%) opinaron, el manejo de las teorías sobre el trastorno disocial escolar proporcionan la habilidad para identificar comportamientos vinculado al trastorno disocial escolar, el cual afecta el desempeño académico estudiantil, aunque un cinco por ciento (5\%) expresó estar en desacuerdo sobre este aspecto. De igual manera, el ítems 4 una gran cantidad de docentes el cincuenta y tres por ciento (53\%) coincidieron, la formación basada en competencias profesionales favorece significativamente al docente en el desarrollo de estrategias pedagógicas que facilitan la prevención del comportamiento disocial en el ambiente escolar, solo un cinco por ciento (5\%) señaló estar en desacuerdo.

Tabla 4: Dimensión: Roles para el manejo del comportamiento disocial escolar

\begin{tabular}{|c|c|c|c|c|c|c|c|c|c|}
\hline \multicolumn{2}{|r|}{ Aspecto a Evaluar } & \multicolumn{2}{|c|}{$\begin{array}{l}\text { Muy de } \\
\text { acuerdo }\end{array}$} & \multicolumn{2}{|c|}{ De acuerdo } & \multicolumn{2}{|c|}{$\begin{array}{l}\text { Ni de acuerdo } \\
\text { ni en } \\
\text { desacuerdo }\end{array}$} & \multicolumn{2}{|c|}{$\begin{array}{c}\text { En } \\
\text { desacuerdo }\end{array}$} \\
\hline Ítems & $\begin{array}{l}\text { Dimensión: Roles para el manejo del } \\
\text { comportamiento disocial escolar. }\end{array}$ & $\mathrm{F}$ & $\%$ & $\mathrm{~F}$ & $\%$ & $F$ & $\%$ & $F$ & $\%$ \\
\hline 5 & $\begin{array}{l}\text { La teoría de la acción docente y el humanismo } \\
\text { permite al docente ejercer con eficacia el rol de } \\
\text { orientación para prevenir la agresividad y la } \\
\text { violencia en la institución educativa. } \\
\text { El modelo de acción docente ejecutado en la }\end{array}$ & 13 & 68 & 5 & 26 & 1 & 5 & 0 & 0 \\
\hline 6 & $\begin{array}{l}\text { institución se ha caracterizado por desmejorar el } \\
\text { comportamiento y el bienestar del estudiante. } \\
\text { El desarrollo de estrategias fundamentadas en el }\end{array}$ & 0 & 0 & 1 & 5 & 2 & 11 & 16 & 84 \\
\hline 7 & $\begin{array}{l}\text { modelo de acción docente ha permitido potenciar el } \\
\text { aprendizaje del estudiante y el desarrollo integral. }\end{array}$ & 14 & 74 & 3 & 16 & 2 & 11 & 0 & 0 \\
\hline 8 & $\begin{array}{l}\text { El modelo implementado no mostraba } \\
\text { correspondencia con el aprendizaje integral y el } \\
\text { desarrollo de la conducta humana en el estudiante. }\end{array}$ & 0 & 0 & 1 & 5 & 1 & 5 & 17 & 89 \\
\hline
\end{tabular}


Los datos recogidos en la Tabla 4, dimensión roles para el manejo del comportamiento disocial escolar, se constató en el ítem 5 , el sesenta y ocho por ciento (68\%) de los docentes opinaron estar muy de acuerdo con la teoría de la acción docente y el humanismo, estos conceptos ayudan al docente desempeñar con eficacia el rol de orientador, prevenir la agresividad y la violencia en la institución educativa y el veintiséis por ciento (26\%) manifestó estar de acuerdo con esta idea. No obstante, en el ítems 6, casi la totalidad de los docentes el ochenta y cuatro por ciento (84\%) exteriorizaron estar en desacuerdo en que el modelo aplicado no se caracterizó por desmejorar el comportamiento y el bienestar del estudiante, a diferencia de un cinco por ciento, (5\%) estuvo de acuerdo sobre este particular. En el ítems 7, el setenta y cuatro \% (74\%) expresó estar muy de acuerdo en que el desarrollo de estrategias fundamentadas en el modelo de acción docente permitió potenciar el aprendizaje y el desarrollo integral del estudiante y un once por ciento (11\%) se ubicó en la opinión ni de acuerdo ni en desacuerdo sobre este ítem. Seguidamente, el ítems 8, el ochenta y nueve por ciento $(89 \%)$ de los docentes la mayoría señalaron estar desacuerdo sobre el mismo, ya que el modelo implementado si mostró correspondencia con el aprendizaje integral y el desarrollo de la conducta humana en el estudiante.

Tabla 5: Dimensión: Roles para el manejo del comportamiento disocial escolar

\begin{tabular}{|c|c|c|c|c|c|c|c|c|c|}
\hline \multirow{2}{*}{\multicolumn{2}{|c|}{$\begin{array}{l}\text { Aspecto a Evaluar } \\
\text { Dimensión: Competencias }\end{array}$}} & \multicolumn{2}{|c|}{$\begin{array}{l}\text { Muy de } \\
\text { acuerdo }\end{array}$} & \multicolumn{2}{|c|}{$\begin{array}{c}\text { De } \\
\text { acuerdo }\end{array}$} & \multicolumn{2}{|c|}{$\begin{array}{c}\text { Ni de } \\
\text { acuerdo ni en } \\
\text { desacuerdo }\end{array}$} & \multicolumn{2}{|c|}{$\begin{array}{c}\text { En } \\
\text { desacuerdo }\end{array}$} \\
\hline & & $F$ & $\%$ & $F$ & $\%$ & $F$ & $\%$ & $F$ & $\%$ \\
\hline 9 & $\begin{array}{l}\text { El uso de las competencias básicas han favorecido el } \\
\text { desempeño docente para identificar comportamientos } \\
\text { disóciales en el estudiante. }\end{array}$ & 14 & 74 & 3 & 16 & 2 & 11 & 0 & 0 \\
\hline 10 & $\begin{array}{l}\text { La aplicabilidad de la competencia profesional mejora la } \\
\text { integración de los padres representantes en el proceso de } \\
\text { formación educativo del joven estudiante }\end{array}$ & 15 & 79 & 3 & 16 & 1 & 5 & 0 & 0 \\
\hline 11 & $\begin{array}{l}\text { El modelo desarrollado ha despertado interés en la } \\
\text { comunidad para ayudar al joven estudiante con problemas } \\
\text { de comportamientos disocial }\end{array}$ & 16 & 84 & 1 & 5 & 1 & 5 & 0 & 0 \\
\hline 12 & $\begin{array}{l}\text { El modelo de acción docente planteado excluye las } \\
\text { necesidades intrínsecas de conducta en el estudiante. }\end{array}$ & 0 & 0 & 1 & 5 & 2 & 11 & 16 & 84 \\
\hline
\end{tabular}

La información reflejada en la Tabla 5, dimensión competencias profesionales se logró verificar lo siguiente: En el ítem 9 los docentes expresaron, un setenta y cuatro por ciento $(74 \%)$ constató estar muy de acuerdo con el uso de las competencias básicas para identificar comportamientos disóciales en el estudiante y un once por ciento (11\%) estuvo ni de acuerdo ni en desacuerdo en correlación a esta idea. De igual manera sucedió con el ítems 10, donde la mayor parte de los docentes, el setenta y nueve por ciento (79\%) coincidieron en que la aplicabilidad de la competencia profesional mejora la integración de los padres representantes en el proceso de formación educativo del joven estudiante, solo un cinco por ciento (5\%) opinó estar ni de acuerdo ni en desacuerdo en la aplicabilidad de la competencia profesional. El ítems 11, la mayoría de los docentes el ochenta y cuatro por ciento (84\%) afirmó estar muy de acuerdo en que el modelo desarrollado ha despertado interés en la comunidad para ayudar al joven estudiante con problemas de comportamientos disocial a diferencia de una pequeña minoría, un cinco por ciento $(5 \%)$ respondió lo contrario. Por último, el ítems 12, la mayoría de los docente un ochenta y cuatro por ciento (84\%) manifestaron estar total en desacuerdo sobre esta pregunta, dado que el modelo de acción docente planteado no excluyó las necesidades intrínsecas de conducta en el estudiante.

\section{EVALUACION DEL IMPACTO SOBRE EL MODELO}

A partir del resultado, la experiencia reflejada en el instrumento de observación tipo Likert empleado por los tres docentes directivos de la Unidad Educativa "Alto Barinas Norte", se pudo inferir un resultado positivo, pues se confirmó la participación y aceptación del modelo por parte de los docentes determinándose así la validez del modelo propuesto. Se fortaleció la acción docente y la formación de competencias mediante los talleres que permitieron al docente orientar el comportamiento disocial escolar Todorescu et al., (2015); Blašková et al., (2014). En consecuencia, se presenta a continuación los siguientes impactos: A nivel institucional, se atendió los lineamientos emanados por el Programa de Protección y Bienestar Estudiantil del Ministerio del Poder Popular para la Educación en Venezuela para ejecutar modelos de intervención como el aquí propuesto Gómez, (2018), que tienen como función proporcionar estrategias pedagógicas que orienten la conducta del estudiante, así como también líneas de acción didácticas en el manejo de situaciones no deseadas en el ámbito escolar. Para Pérez, (2011); Contreras, (2009), la capacitación a los docentes sobre los tipos de roles y de orientador, trajo como resultado la elaboración de un cronograma de reuniones 
permanentes para el intercambio de experiencias que facilite la incorporación de contenidos en la planificación, relacionados con las competencias para el desempeño docente y de orientador. De esta manera se consolidó las habilidades, destrezas propias para el consecuente efecto del comportamiento del estudiante en el ambiente escolar.

A nivel del docente, se logró realizar intercambios entre docentes de otras instituciones a fin de proyectar la experiencia obtenida, Ornelas et al., (2012); Marinho-Araujo y Almeida, (2016). Este logro pudo servir de motivación para impulsar a otras instituciones a fin de prevenir este tipo de comportamiento relacionados con el trastorno disocial escolar. La experiencia adquirida durante los talleres permitió al docente expresar estilos de vida saludables que pueden conducir el comportamiento del estudiante hacia el logro de metas determinadas que contribuyen con el desarrollo cognitivo y personal del estudiante. Se consiguió planificar jornadas, encuentros, seminarios como estrategia que apoyo al docente mantenerse en constante proceso de actualización. En efecto, la construcción epistemológica fundamentada la teoría de en Carl. R. Rogers, permitió resaltar las potencialidades y la autenticidad genuina del docente para orientar la conducta estudiantil, Vieira y Pinheiro, (2013); Dutra et al., (2016); Bryan et al., (2015). Además se generó en el docente procesos de autoevaluación, coevaluación como estrategia autocritica fundamentadas en la experiencia, permitan mejorar de manera consecutiva la acción docente y el aprendizaje establecido en las competencias. En la familia y el estudiante, Smolkowski, et al., (2017), la familia y la institución educativa, son las responsables en proporcionar al educando la formación de patrones de comportamientos cónsonos con las normas de convivencia y la prevención de conductas de riesgo. Sobre esta premisa, se logró valorar a la familia como base fundamental para promover en el aula de clase el crecimiento cognitivo, afectivo del estudiante, como un ser social, racional, participativo, creativo, espontáneo que genera experiencias significativas de aprendizaje, situaciones que contribuyen con el desarrollo pleno de sus potencialidades humanas y los valores de convivencia escolar.

\section{CONCLUSIONES}

De acuerdo a los resultados y su discusión se pueden obtener las siguientes conclusiones principales:

1.- El modelo de acción tuvo como impacto principal fortalecer el desempeño docente en el ambiente escolar y mejoró las competencias en los profesores para prevenir situaciones futuras relacionadas con los comportamientos vinculados con el trastorno disocial escolar. El modelo ejecutado sirvió de referencia para otras instituciones educativas ajustado a necesidades específicas gracias al intercambio de experiencias positivas.

2. El modelo ejecutado constituyó una innovación educativa, dado que fue fácil su puesta en práctica de las fases, los talleres proporcionó al docente las experiencias y procedimentales para orientar los comportamiento disóciales.

3.- Los impactos obtenidos durante la ejecución del modelo de acción docente, fue comparado con otros modelos pedagógicos el cual beneficia directamente a los estudiantes y docentes de la institución. Esto permitió retomar los lineamientos emanados por el Programa de Protección y Bienestar Estudiantil del Ministerio del Poder Popular para la Educación en Venezuela.

\section{REFERENCIAS}

Aarón, M.A., P. E. Choles y A.D. Solano, Representación del Proceso Formativo de una institución Etnoeducativa a través de la Técnica del Modelo Gráfico y Descriptivo usando Diagramas de influencia y de Forrester, doi: 10.4067/S071807642016000300008, Información Tecnológica, 27(3), 81-92 (2016)

Abbott, J.A., H.D. Soifer y M. Vom Hau, Transforming the Nation? The Bolivarian Education Reform in Venezuela, doi: 10.1017/S0022216X17000402, Journal of Latin American Studies, 49(4), 885-916 (2017)

Acevedo, D., S. Cavadia y A. Alvis, Estilos de aprendizaje de los estudiantes de la Facultad de Ingeniería de la Universidad de Cartagena, Colombia, doi: 10.4067/S0718-50062015000400003, Formación Universitaria, 8(4), 15-22 (2015)

Almeida, F.N.D., For a general theory of health: preliminary epistemological and anthropological notes, doi: 10.1590/S0102-311X2001000400002, Cadernos de Saúde Pública, 17(4), 753-770 (2001)

Bastos, J. L., R. P. Duquia, D.A. González-Chica, J. M. Mesa y R. R. Bonamigo, Field work I: selecting the instrument for data collection, doi: 10.1590/abd1806-4841.20143884, Anais brasileiros de dermatologia, 89(6), 918-923 (2014)

Blašková, M., R. Blaško, M. Jankalová y R. Jankal, Key personality competences of university teacher: comparison of requirements defined by teachers and/versus defined by students, doi: 10.1016/j.sbspro.2013.12.731, Procedia-Social and Behavioral Sciences, 114, 466-475 (2014) 
Bontempi Jr, B. y E.D. Pietri, Educative processes and the formation of individuals today, doi: 10.1590/s151797022017430200, Educação e Pesquisa, 43(2), 301-309 (2017)

Bradfield, R., G. Cairns y G. Wright, Teaching scenario analysis-an action learning pedagogy, doi: 10.1016/j.techfore.2015.05.005, Technological Forecasting and Social Change, 100, 44-52 (2015)

Bryan, V.D., J. Lindo, P. Anderson-Johnson y S. Weaver, Using Carl Rogers' Person-Centered Model to Explain Interpersonal Relationships at a School of Nursing, doi: 10.1016/j.profnurs.2014.07.003, Journal of Professional Nursing, 31(2), 141-148 (2015)

Carvalho, R.G. y R.F. Novo, The relationship between structural dimensions of personality and school life in adolescence, doi: 10.1590/1678-7153.201427218, Psicologia: Reflexão e Crítica, 27(2), 368-376 (2014)

Cheon, S. H., J. Reeve y N. Ntoumanis, A needs-supportive intervention to help PE teachers enhance students' prosocial behavior and diminish antisocial behavior, doi: 10.1016/j.psychsport.2017.11.010, Psychology of Sport and Exercise, 35, 74-88 (2018)

Cid, J. E., L. P. Cuadra, S. H. Cuevas y A. A. Villalobos, Articulación Educación y Trabajo: Un estudio desde la Mirada de los Docentes de la Educación Técnica Regional Chilena y sus Necesidades de Perfeccionamiento Pedagógico, doi: 10.4067/S0718-07642017000100004, Información Tecnológica, 28(1), 25-34 (2017)

Contreras, D.L.M., Orientación educativa: práctica de intervención permanente en los centros escolares, E-ISSN: 16962095, Rev. Redalyc, 7(19), 1449- 1460 (2009)

Flores, F. J., J. E. Peinado, M. Ornelas y L. López, Composición Factorial de una Escala Autoeficacia en Conductas de Cuidado de la Salud en Estudiantes de Ingeniería, doi: 10.4067/S0718-50062012000300006, Formación Universitaria, 5(3), 43-54 (2012)

García Valdés, M. y M. M. Suárez, El método Delphi para la consulta a expertos en la investigación científica, ISSN: 08643466, Revista Cubana de Salud Pública, 39(2), 253-267 (2013)

Gómez, J., Modelo de Acción Docente como Alternativa Pedagógica para el Manejo del Trastorno Disocial Escolar, 5362, Información Tecnológica, 29(2), 53-62 (2018)

Hernández, R. C. Fernández y P. Baptista, Metodología de la Investigación, 6ª Ed., 270-299, Mc Graw Hill, D.F., México (2014) Jorm, A.F., Using the Delphi expert consensus method in mental health research. doi: 10.1177/0004867415600891, Australian \& New Zealand Journal of Psychiatry, 49(10), 887-897 (2015)

Ley Orgánica de Educación, Gaceta Oficial de la República Bolivariana de Venezuela, 5929 (Extraordinaria), Agosto 15 (2009)

Lima, R.M., J. Dinis-Carvalho, R.M. Sousa, P. Arezes y D. Mesquita, Development of competences while solving real industrial interdisciplinary problems: a successful cooperation with industry, doi:10.1590/0103-6513.230016, Production, 27, SPE (2017)

Marinho-Araujo, C.M. y L.S. Almeida, Approach to competences, human development and higher education, doi: 10.1590/0102-3772e32ne212, Psicologia: Teoria e Pesquisa, 32, SPE (2016)

Méndez-Garrido, J.M. y P.M. Monescillo, Estrategias para la evaluación de programas de orientación, XXI, Revista de Educación, 181-202 (2009)

Ornelas, M., H. Blanco, G. Gastélum y A. Chávez, Autoeficacia percibida en la conducta académica de estudiantes universitarias, doi: 10.4067/S0718-50062012000200003, Formación Universitaria, 5(2), 17-26 (2012)

Palella S. y F. Martins. Metodología de la Investigación Cuantitativa, Caracas, Venezuela, FEDUPEL (2012)

Pérez Solís, M., ¿Puede un psicopedagogo desempeñar el rol del psicólogo educativo? Profesionalización frente al intrusismo profesional, Estud. Psicol, Campinas, 28(1), 3-13 (2011)

Piotrowska, P.J., C.B. Stride, S.E. Croft y R. Rowe, Socioeconomic status and antisocial behaviour among children and adolescents: A systematic review and meta-analysis, doi: 10.1016/j.cpr.2014.11.003, Clinical psychology review, 35, 4755 (2015)

Rosa, G., G. Riberas, L. Navarro-Segura y J. Vilar, El coaching como herramienta de trabajo de la competencia emocional en la formación de estudiantes de educación social y trabajo social de la Universidad Ramón Llull, España, doi.org/10.4067/S0718-50062015000500009, Formación Universitaria, 8(5), 77-90 (2015)

Smolkowski, K., J.R. Seeley y otros cinco autores, Effectiveness evaluation of the Positive Family Support intervention: A three-tiered public health delivery model for middle schools, doi: 10.1016/j.jsp.2017.03.004, Journal of school psychology, 62, 103-125 (2017)

Todorescu, L. L., M. M. Popescu-Mitroi y A. Greculescu. Students' Views on the Teacher as An Information Provider, doi: 10.1016/j.sbspro.2015.07.121, Procedia-Social and Behavioral Sciences, 197, 184-189 (2015)

Urazán-Torres, G. R., M. J. Puche-Cabrera, M. Caballero-Forero y C. A. Rey-Anacona, Cognitive and Executive Functions in Colombian School Children with Conduct Disorder: Sex Differences, doi; 10.1016/S0034-7450(13)70029-5, Rev. Colombiana de Psiquiatría, 42(4), 324-332 (2013)

Urhahne, D., Teacher behavior as a mediator of the relationship between teacher judgment and students' motivation and emotion, doi: 0.1016/j.tate.2014.09.006, Teaching and Teacher Education, 45, 73-82 (2015) 
Vera, G.D., D. Jiménez y A. Barreto, Contemporary Public Policy on School-Based Guidance and Counseling in Venezuela, Costa Rica, and Other Latin American Countries, doi: 10.1007/978-3-319-58179-8_31, In International Handbook for Policy Research on School-Based Counseling, 473-483 (2017)

Vera, P., J. Ezpeleta y R. Granero, Universidad Autónoma de Barcelona, Conducta antisocial: asociación con psicopatología en niños y adolescentes de México, Departamento de Psicología Clínica de la Salud, 45, s124-s131 (2013)

Vieira, E.M. y F. Pinheiro, Person centered psychotherapy: an encounter with oneself or a confrontation with the other? doi: 10.1590/S0103-166X2013000200009, Estudos de Psicologia Campinas, 30(2), 231-238 (2013)

Wang, M.T. y J.A. Fredricks, The reciprocal links between school engagement, youth problem behaviors, and school dropout during adolescence, doi: 10.1111 / cdev.12138, Child development, 85(2), $722-737$ (2014)

Zalyaeva, E.O. e I.M. Solodkova, Teacher-student collaboration: Institute of economics and finance Kazan federal university approach, doi:10.1016/j.sbspro.2014.09.271, Procedia-Social and Behavioral Sciences, 152, 1039-1044 (2014) 